\title{
Testing Statistical Hypotheses Based on Fuzzy Confidence Intervals
}

\author{
Jalal Chachi ${ }^{1}$, Seyed Mahmoud Taheri ${ }^{1}$ and Reinhard Viertl ${ }^{2}$ \\ ${ }^{1}$ Isfahan University of Technology, Iran \\ ${ }^{2}$ Technische Universität Wien, Austria
}

\begin{abstract}
A fuzzy test for testing statistical hypotheses about an imprecise parameter is proposed for the case when the available data are also imprecise. The proposed method is based on the relationship between the acceptance region of statistical tests at level $\beta$ and confidence intervals for the parameter of interest at confidence level $1-\beta$. First, a fuzzy confidence interval is constructed for the fuzzy parameter of interest. Then, using such a fuzzy confidence interval, a fuzzy test function is constructed. The obtained fuzzy test, contrary to the classical approach, leads not to a binary decision (i.e. to reject or to accept the given null hypothesis) but to a fuzzy decision showing the degrees of acceptability of the null and alternative hypotheses. Numerical examples are given to demonstrate the theoretical results, and show the possible applications in testing hypotheses based on fuzzy observations.
\end{abstract}

Zusammenfassung: Aufbauend auf der Beziehung zwischen Konfidenzintervallen für Parameter von stochastischen Modellen und statistischen Tests für Parameterhypothesen, wird eine Verallgemeinerung für den Fall unscharfer Daten und zugehörigen unscharfen Konfidenzintervallen vorgeschlagen. Die zugehörigen verallgemeinerten Tests liefern unscharfe Entscheidungen mit Graden von Annahmen bzw. Ablehungen von Hypothesen. Numerische Beispiele aus der Lebensdaueranalyse zeigen die Anwendbarkeit von solchen statistischen Tests für unscharfe Beobachtungen.

Keywords: Confidence Interval, Fuzzy Parameter, Fuzzy Test, Fuzzy Random Variable, Lifetime Testing, Testing Statistical Hypotheses.

\section{Introduction}

Hypothesis testing and confidence intervals play a prominent role in classical statistical texts. In the classical theory of parametric statistical inference there is a relationship between the totality of parameter values for which the null hypothesis is accepted and the structure of the confidence intervals. Namely, a family of acceptance regions for a statistical test about a parameter $\theta$, at level $\beta$, is equivalent to a certain family of confidence intervals for the parameter, at confidence level $1-\beta$. If the value of the parameter specified by the null hypothesis is contained in the $1-\beta$ confidence interval then the null hypothesis cannot be rejected at level $\beta$, and if it is not contained in the $1-\beta$ confidence interval then the null hypothesis can be rejected at level $\beta$.

As an early study, we can mention the work by Press (1966), in which he compared two well known procedures proposed for the Behrens-Fisher problem, based on comparing the expected length of the confidence intervals they yield. Finner (1994), based on the 
duality between tests and confidence sets, introduced a method to derive one-sided confidence bounds following the rejection of a null hypothesis with two-sided alternatives. Bolviken and Skovlund (1996) investigated the structure and characteristics of confidence intervals obtained by inverting some Monte Carlo tests. Dümbgen (1998) introduced some goodness-of-fit tests and studied their application to nonparametric confidence sets. Holm (1999) by introducing the concept of confidence directional sets, studied the construction of multiple confidence sets related to stage-wise multiple tests. Mukerjee and Reid (2001) compared some test statistics via expected lengths of associated confidence intervals based on their asymptotic behaviors. The topic of relation between testing hypotheses and confidence intervals has been studied also from a practical point of view. For example, Brandstätter and Kepler (1999) argued to replace significance testing by confidence intervals in psychological methodology. Tryon (2001) described an integrated, alternative inferential confidence interval approach to testing for statistical difference, equivalence, and indeterminacy that is algebraically equivalent to standard testing statistical hypotheses. Beaulieu-Préost (2006) provided the conceptual tools necessary to implement an approach based on confidence intervals, and demonstrated why such an approach is an interesting alternative to an approach based on hypothesis testing. For a brief technical review on the relationship between testing hypotheses and confidence intervals, the reader is referred to Casella and Berger (2002, p. 444) and Lehmann and Romano (2005, p. 72).

In this paper, we wish to apply this point of view to fuzzy environment to propose a fuzzy test for testing hypotheses about a fuzzy parameter of a statistical model, based on a fuzzy confidence interval for the fuzzy parameter. The problems of testing fuzzy hypotheses and fuzzy confidence intervals have been devised independently by many authors. But, up till now, these two problems, i.e. testing statistical hypotheses and confidence intervals in fuzzy environment, have been separately considered in the literature. Some of the main approaches to such problems are briefly reviewed below.

Grzegorzewski and Hryniewicz (1997) reviewed some methods in testing statistical hypotheses in fuzzy environment, pointing out their advantages or disadvantages and practical problems. Grzegorzewski $(2000,2009)$ suggested some fuzzy tests for testing statistical hypotheses based on vague data in parametric and non-parametric populations. Arnold and Gerke (2003) studied testing fuzzy linear hypotheses in linear regression models. Filzmoser and Viertl (2004) presented an approach for testing hypotheses at the basis of fuzzy values by introducing the fuzzy $p$-value, (see also Parchami, Taheri, and Mashinchi, 2010 for a $p$-value based approach to the problem of testing fuzzy hypotheses). Montenegro, Colubi, Casals, and Gil (2004), using a generalized metric for fuzzy numbers, proposed a method to test the fuzzy mean of a fuzzy random variable (abbreviated as FRV). Parchami, Mashinchi, Yavari, and Maleki (2005) studied a fuzzy version of some process capability indices when specification limits are fuzzy rather than precise, and obtained fuzzy confidence intervals for such indices. Wu (2005) proposed decision rules based on FRVs that are used to accept or reject the null and alternative hypotheses about a fuzzy parameter using the concepts of degrees of optimism and pessimism. GonzálezRodríguez, Montenegro, Colubi, and Gil (2006b) introduced a bootstrap approach to the one-sample test of mean for imprecisely valued sample data. Hryniewicz (2006) investigated the problem of the interpretation of the results of statistical tests in terms of the 
theory of possibility. In this work, the concept of $p$-value was given a new possibilistic interpretation and was generalized for the case of imprecisely defined statistical hypotheses and vague statistical data. Viertl $(2006,2011)$ investigated some methods to construct confidence intervals and statistical tests for fuzzy data. Akbari and Rezaei (2009) investigated a bootstrap method for inference about the variance based on fuzzy data. Wu (2009) and Chachi and Taheri (2011) proposed some approaches to construct fuzzy confidence intervals for the unknown fuzzy parameter. Arefi and Taheri (2011) developed an approach to test fuzzy hypotheses upon a fuzzy test statistic for vague data. GonzálezRodríguez, Colubi, and Gil (2012) developed a one-way ANOVA test approach for fuzzy observations in which the fuzzy observations are treated as functional data of a functional Hilbert space. The reader is referred to the work by Taheri (2003) for a general review on statistical methods in fuzzy environment.

The aim of this work is to introduce a new approach to the problem of testing statistical hypotheses for fuzzy data using the relationship between confidence intervals and testing hypotheses. To do this we employ the method of constructing fuzzy confidence intervals for fuzzy parameters investigated by Chachi and Taheri (2011).

The rest of this paper is organized as follows. In the next section, some basic concepts that will be used in the sequel are recalled. Sections 3 and 4 provide statement and formalization of the problem of constructing fuzzy tests for vague data and imprecise parameters, respectively. Numerical examples are given in Section 5 to clarify the theoretical results, and to show possible applications of testing hypotheses about a fuzzy parameter based on fuzzy observations. In the final section, we make some concluding remarks.

\section{Preliminaries}

\subsection{Fuzzy Arithmetic}

In this paper let $\mathbb{R}$, the set of all real numbers, be the universal set which is endowed with a topological structure. A fuzzy subset (briefly, a fuzzy set) $\tilde{A}$ of $\mathbb{R}$ is defined by its membership function $\tilde{A}: \mathbb{R} \rightarrow[0,1]$. For each $\alpha \in(0,1]$, the $\alpha$-level set of $\tilde{A}$ is defined by $\tilde{A}_{\alpha}=\{x \in \mathbb{R}: \tilde{A}(x) \geq \alpha\}$, and $\tilde{A}_{0}$ is the closure of the set $\{x \in \mathbb{R}: \tilde{A}(x)>0\}$. The fuzzy set $\tilde{A}$ is called a fuzzy number if each $\tilde{A}_{\alpha}$ is a nonempty closed bounded interval for all $\alpha \in(0,1]$. The $\alpha$-level set of each fuzzy number $\tilde{A}$ is usually denoted by $\tilde{A}_{\alpha}=$ $\left[A_{\alpha}^{l}, A_{\alpha}^{u}\right]$, where $A_{\alpha}^{l}=\inf \{x \in \mathbb{R}: A(x) \geq \alpha\}$ and $A_{\alpha}^{u}=\sup \{x \in \mathbb{R}: A(x) \geq \alpha\}$.

A wide class of fuzzy sets in $\mathcal{F}(\mathbb{R})$ (the set of all fuzzy numbers of $\mathbb{R}$ ), which is rich and flexible enough to cover most of the applications, is the class of so-called $L R$-fuzzy numbers $\tilde{N}=(n, l, r)_{L R}$ with central value $n \in \mathbb{R}$, left and right spreads $l, r \in \mathbb{R}$, decreasing left and right shape functions $L, R: \mathbb{R}^{+} \rightarrow[0,1]$, with $L(0)=R(0)=1$. Typically, the $L R$-fuzzy number $\tilde{N}$ could be shown by the following membership function (Zimmermann, 2001)

$$
\tilde{N}(x)= \begin{cases}L\left(\frac{n-x}{l}\right) & \text { if } x \leq n \\ R\left(\frac{x-n}{r}\right) & \text { if } x>n\end{cases}
$$


We can easily obtain the $\alpha$-level sets of $\tilde{N}$ as

$$
\tilde{N}_{\alpha}=\left[N_{\alpha}^{l}, N_{\alpha}^{u}\right]=\left[n-L^{-1}(\alpha) l, n+R^{-1}(\alpha) r\right], \quad \forall \alpha \in[0,1] .
$$

A special kind of $L R$-fuzzy numbers are the triangular fuzzy numbers denoted by $\tilde{A}=$ $\left(a, a_{l}, a_{r}\right)_{T}$. The membership function and the $\alpha$-level sets of a triangular fuzzy number $\tilde{A}$ are

$$
\begin{aligned}
\tilde{A}(x) & = \begin{cases}\frac{x-\left(a-a_{l}\right)}{a} l_{1} & \text { if } a-a_{l} \leq x<a, \\
\frac{\left(a+a_{r}\right)-x}{a} r_{r} & \text { if } a \leq x \leq a+a_{r},\end{cases} \\
\tilde{A}_{\alpha} & =\left[A_{\alpha}^{l}, A_{\alpha}^{u}\right]=\left[a-(1-\alpha) a_{l}, a+(1-\alpha) a_{r}\right], \quad \forall \alpha \in[0,1] .
\end{aligned}
$$

Using the extension principle, arithmetic operations on fuzzy numbers are defined by

$$
(\tilde{A} \bigcirc \tilde{B})(z)=\sup _{x, y: x \circ y=z} \min \{\tilde{A}(x), \tilde{B}(y)\},
$$

where $\bigcirc$ is any kind of the extended arithmetic operations $\oplus, \ominus, \otimes$, and $\oslash$, and $\circ$ is any kind of the arithmetic operations,,$+- \times$, and /. It is well-known that if $\tilde{A}$ and $\tilde{B}$ are two fuzzy numbers, then $\tilde{A} \oplus \tilde{B}$ is also a fuzzy number and $(\tilde{A} \oplus \tilde{B})_{\alpha}=\left[A_{\alpha}^{l}+B_{\alpha}^{l}, A_{\alpha}^{u}+B_{\alpha}^{u}\right]$, for all $\alpha \in(0,1]$ (for more details, see e.g. (Zimmermann, 2001)).

A well-known ordering of fuzzy numbers, used in the sections below for defining hypotheses about a fuzzy parameter, is defined as (Wu, 2005):

1. $\tilde{A}=(\neq) \tilde{B}$, if $A_{\alpha}^{l}=(\neq) B_{\alpha}^{l}$ and $A_{\alpha}^{u}=(\neq) B_{\alpha}^{u}$ for any $\alpha \in(0,1]$.

2. $\tilde{A} \preceq(\prec) \tilde{B}$, if $A_{\alpha}^{l} \leq(<) B_{\alpha}^{l}$ and $A_{\alpha}^{u} \leq(<) B_{\alpha}^{u}$ for any $\alpha \in(0,1]$.

3. $\tilde{A} \succeq(\succ) \tilde{B}$, if $A_{\alpha}^{l} \geq(>) B_{\alpha}^{l}$ and $A_{\alpha}^{u} \geq(>) B_{\alpha}^{u}$ for any $\alpha \in(0,1]$.

\subsection{Fuzzy Random Variables (FRVs)}

Let $(\Omega, \mathcal{A})$ be a measurable space where $\Omega$ is a set of all possible outcomes of an experiment and $\mathcal{A}$ is a $\sigma$-algebra of subsets of $\Omega$ (the set of all possible events) and $(\mathbb{R}, \mathcal{B})$ be the Borel measurable space. Throughout this paper, we assume that all random variables have the same probability space $(\Omega, \mathcal{A}, P)$, where $P$ is a probability measure on the measurable space $(\Omega, \mathcal{A})$.

The set valued function $f: \Omega \rightarrow \mathcal{P}(\mathbb{R})$ (where $\mathcal{P}(\mathbb{R})$ is the power set of $\mathbb{R}$ ) is called measurable if and only if $\{(x, y): y \in f(x)\}$ is $\mathcal{A} \times \mathcal{B}$-measurable (Aumann, 1965). If $\tilde{f}: \Omega \rightarrow \mathcal{F}(\mathbb{R})$ is a fuzzy-valued function then $\tilde{f}_{\alpha}$ is a set-valued function for all $\alpha \in[0,1]$ where $\tilde{f}_{\alpha}(\omega)=\{x \in \mathbb{R} \mid \tilde{f}(\omega)(x) \geqslant \alpha\}$ (Nguyen, 2006). The fuzzy-valued function $\tilde{f}$ is called measurable if and only if $\tilde{f}_{\alpha}$ is (set-valued) measurable for all $\alpha \in[0,1]$. The fuzzy-valued function $\mathcal{X}: \Omega \rightarrow \mathcal{F}(\mathbb{R})$ is called a FRV if $\mathcal{X}$ is measurable. It is shown that, the fuzzy-valued function $\mathcal{X}: \Omega \rightarrow \mathcal{F}(\mathbb{R})$ is a FRV if and only if $X_{\alpha}^{l}: \Omega \rightarrow \mathbb{R}$ and $X_{\alpha}^{u}: \Omega \rightarrow \mathbb{R}$ are two real valued random variables for all $\alpha \in(0,1]$ (where $\forall \omega \in$ $\Omega ; \mathcal{X}(\omega)_{\alpha}=\left[X_{\alpha}^{l}(\omega), X_{\alpha}^{u}(\omega)\right]$ ) (Puri and Ralescu, 1986; Kruse and Meyer, 1987). 
A fuzzy number $\mathcal{E}(\mathcal{X}) \in \mathcal{F}(\mathbb{R})$ is called the (fuzzy) expectation of $\mathcal{X}$, if $\mathcal{E}(\mathcal{X})_{\alpha}=$ $\left[E X_{\alpha}^{l}, E X_{\alpha}^{u}\right]$ for all $\alpha \in(0,1]$. It is proved that under some conditions, $\mathcal{E}(\mathcal{X})$ exists and it is a unique fuzzy number in $\mathcal{F}(\mathbb{R})$ (Feng, 2000).

A FRV $\mathcal{X}$ is called a normal (Gaussian) FRV if $\mathcal{X}=\mathcal{E}(\mathcal{X}) \oplus \Xi$, where $\Xi$ is a (usual) normal random variable with mean 0 and variance $\sigma^{2}$, i.e. $\Xi \sim N\left(0, \sigma^{2}\right)$ (Puri and Ralescu, 1985). For a normal FRV $\mathcal{X}$, we have $X_{\alpha}^{l} \sim N\left(E X_{\alpha}^{l}, \sigma^{2}\right)$ and $X_{\alpha}^{u} \sim$ $N\left(E X_{\alpha}^{u}, \sigma^{2}\right)$, for all $\alpha \in(0,1]$ (Feng, 2000). Let $\tilde{\theta}=\mathcal{E}(\mathcal{X})$. Then we say that $\mathcal{X}$ is distributed as $\mathcal{N}\left(\tilde{\theta}, \sigma^{2}\right)$, and write $\mathcal{X} \sim \mathcal{N}\left(\tilde{\theta}, \sigma^{2}\right)$, if $\mathcal{X}$ is a normal FRV with $\mathcal{X}=\mathcal{E}(\mathcal{X}) \oplus \Xi=\tilde{\theta} \oplus \Xi(\mathrm{Wu}, 2005)$.

FRVs $\mathcal{X}$ and $\mathcal{Y}$ are called identically distributed if $X_{\alpha}^{l}$ and $Y_{\alpha}^{l}$, and $X_{\alpha}^{u}$ and $Y_{\alpha}^{u}$ are identically distributed, for all $\alpha \in(0,1]$. They are called independent if each random variable in the set $\left\{X_{\alpha}^{l}, X_{\alpha}^{u}: \alpha \in(0,1]\right\}$ is independent of each random variable in the set $\left\{Y_{\alpha}^{l}, Y_{\alpha}^{u}: \alpha \in(0,1]\right\}$ (Kruse and Meyer, 1987; Wu, 2005, 2009).

We say that $\underline{\mathcal{X}}=\left(\mathcal{X}_{1}, \ldots, \mathcal{X}_{n}\right)$ is a normal fuzzy random sample of size $n$, if the $\mathcal{X}_{i}$ 's are independent and identically distributed normal FRVs, for all $i=1, \ldots, n$. In this case we write $\left(\mathcal{X}_{1}, \ldots, \mathcal{X}_{n}\right) \stackrel{i i d}{\sim} N\left(\tilde{\theta}, \sigma^{2}\right)$. Let $\left(\mathcal{X}_{1}, \ldots, \mathcal{X}_{n}\right) \stackrel{i i d}{\sim} N\left(\tilde{\theta}, \sigma^{2}\right)$, then $\left(X_{1 \alpha}^{l}, \ldots, X_{n \alpha}^{l}\right) \stackrel{i i d}{\sim}$ $N\left(\theta_{\alpha}^{l}, \sigma^{2}\right)$ and $\left(X_{1 \alpha}^{u}, \ldots, X_{n \alpha}^{u}\right) \stackrel{i i d}{\sim} N\left(\theta_{\alpha}^{u}, \sigma^{2}\right)$ for all $\alpha \in(0,1]$, where $\tilde{\theta}_{\alpha}=\left[\theta_{\alpha}^{l}, \theta_{\alpha}^{u}\right](\mathrm{Wu}$, 2005, 2009).

\section{Statement of the Main Problem}

In this paper, by developing the concept of fuzzy confidence intervals and using the oneto-one relationship between tests and confidence intervals, it may be convenient to define fuzzy statistical tests based on fuzzy confidence intervals. Therefore, the results of a fuzzy statistical test can alternatively be stated in terms of the corresponding fuzzy confidence interval. In such a case, we may use, for example, the degree of membership of the null hypothesized fuzzy parameter in the fuzzy confidence interval as the degree of acceptability of the null hypothesis.

In the following, first, we briefly review the correspondence between confidence intervals and testing hypotheses in classical statistical inference (Lehmann and Romano, 2005, Chapter 3). Then, we use such a relationship in fuzzy environment, when the observations and the parameter of interest are fuzzy rather than crisp.

\subsection{The Relationship between Testing Hypotheses and Confidence Intervals: The Classical Approach}

Consider the problem of testing the null hypothesis $H\left(\theta_{0}\right): \theta=\theta_{0}$ versus an alternative hypothesis. For each $\theta_{0}$, let $A\left(\theta_{0}\right)$ denotes the acceptance region of a level $\beta$ test of the hypothesis $H\left(\theta_{0}\right): \theta=\theta_{0}$. If $S(\mathbf{x})=\{\theta: \mathbf{x} \in A(\theta)\}$ then

$$
\theta \in S(\mathbf{x}) \Longleftrightarrow \mathbf{x} \in A(\theta)
$$

and hence

$$
P_{\theta}\{\theta \in S(\mathbf{X})\} \geq 1-\beta, \quad \text { for all } \theta
$$


Thus, based on the relation (1), any family of level $\beta$ acceptance regions leads to a family of confidence sets at confidence level $1-\beta$. Conversely, given any class of confidence sets $S(\mathbf{x})$ satisfying (2), let $A(\theta)=\{\mathbf{x}: \theta \in S(\mathbf{x})\}$. Then the sets $A\left(\theta_{0}\right)$ are level $\beta$ acceptance regions for testing the hypothesis $H\left(\theta_{0}\right): \theta=\theta_{0}$, and the confidence sets $S(\mathbf{x})$ show for each $\theta_{0}$ whether for the particular observed $\mathbf{x}$ the hypothesis $H\left(\theta_{0}\right)$ is accepted or rejected at level $\beta$.

So, a confidence set can be viewed as a statement about testing hypothesis $H(\theta)$, which exhibits the values for which the hypothesis is accepted, i.e. $\{\theta: \theta \in S(\mathbf{x})\}$ (or $\left\{\theta: I_{S(\mathbf{x})}(\theta)=1\right\}$, where $I_{A}(\cdot)$ stands for the indicator function of a set $A$ ) and those for which it is rejected, i.e. $\{\theta: \theta \notin S(\mathbf{x})\}$ (or $\left\{\theta: 1-I_{S(\mathbf{x})}(\theta)=1\right\}$ ). Therefore, we can summarize the test function for testing hypothesis $H_{0}\left(\theta_{0}\right): \theta=\theta_{0}$ versus some $H_{1}$ as

$$
\varphi\left(\mathbf{x} ; \theta_{0}\right)=\left\{\begin{array}{l}
0 \text { if } \theta_{0} \in S(\mathbf{x})\left(\text { or } I_{S(\mathbf{x})}\left(\theta_{0}\right)=1\right), \\
1 \text { if } \theta_{0} \notin S(\mathbf{x})\left(\text { or } I_{S(\mathbf{x})}\left(\theta_{0}\right)=0\right),
\end{array}\right.
$$

where, " 0 " and " 1 " stand for acceptance and rejection of the null hypothesis, respectively. The above test function is a rule stating that the null hypothesis can be rejected if the confidence interval does not contain the hypothesized value of the parameter, and cannot be rejected if the interval contains the hypothesized value.

Example 1. Let $X_{1}, \ldots, X_{n}$ be iid from the normal distribution $N(\theta, 1)$ with an unknown mean $\theta$. A confidence interval at confidence level $1-\beta$ for $\theta$ of the form $S(\boldsymbol{X})=[\bar{X}-$ $\left.\frac{1}{\sqrt{n}} z_{1-\frac{\beta}{2}}, \bar{X}+\frac{1}{\sqrt{n}} z_{1-\frac{\beta}{2}}\right]$ can easily be derived, where $z_{\beta}$ is the $\beta$-quantile of the standard normal distribution, i.e. $\Phi\left(z_{\beta}\right)=\beta$. Assume in a random sample with size $n=25$, $\bar{x}=0.75$ is observed and we want to test $H_{0}: \theta=0.5$ versus $H_{1}: \theta \neq 0.5$ at level $\beta=0.05$. Since $S(\boldsymbol{x})=[0.358,1.142]$, therefore, the test function is derived as

$$
\varphi(\boldsymbol{x} ; 0.5)= \begin{cases}0 & \text { if } I_{S(\boldsymbol{x})}(0.5)=1 \\ 1 & \text { if } I_{S(\boldsymbol{x})}(0.5)=0\end{cases}
$$

In this case, based on the observed value of $\bar{x}$, we accept the null hypothesis at level $\beta=0.05$.

\subsection{The Relationship between Testing Hypotheses and Confidence Intervals: The Fuzzy Environment}

Now, we are going to use the relationship between confidence intervals and testing hypotheses in the case where the parameter of interest as well as available data are fuzzy, and hypotheses of interest are statements about an imprecise parameter. In this case, as we shall see, the fuzzy confidence set can be viewed as a statement about testing the hypothesis $H: \tilde{\theta}=\tilde{\theta}_{0}$, which exhibits the values for which the hypothesis is accepted with degree $C\left(\tilde{\theta}_{0}\right)$, i.e. $\left\{\tilde{\theta}_{0} \in \tilde{C}(\underline{\mathcal{X}}): C\left(\tilde{\theta}_{0}\right)>0\right\}$ and those for which it is rejected with degree $1-C\left(\tilde{\theta}_{0}\right)$, i.e. $\left\{\tilde{\theta}_{0} \in \tilde{C}(\underline{\mathcal{X}}): 1-C\left(\tilde{\theta}_{0}\right)>0\right\}$, where $\tilde{C}(\underline{\mathcal{X}})$ is a fuzzy confidence interval for the fuzzy parameter of interest (Chachi and Taheri, 2011).

In this paper, we will introduce some procedures for testing the following hypotheses:

1. $H_{0}: \tilde{\theta}=\tilde{\theta}_{0}$ versus $H_{1}: \tilde{\theta} \neq \tilde{\theta}_{0}$. 
2. $H_{0}: \tilde{\theta}=\tilde{\theta}_{0}$ versus $H_{1}: \tilde{\theta} \succ \tilde{\theta}_{0}$.

3. $H_{0}: \tilde{\theta}=\tilde{\theta}_{0}$ versus $H_{1}: \tilde{\theta} \prec \tilde{\theta}_{0}$.

Note that, in the above framework, the possible values of the parameter of interest are expressed as linguistic variables, which may be treated as fuzzy perceptions of the usual unknown parameter $\theta$ (Kruse and Meyer, 1987; Grzegorzewski, 2009).

\section{The Proposed Procedure}

In this section, based on fuzzy confidence intervals, we investigate some procedures to provide fuzzy tests for testing hypotheses of a fuzzy parameter for fuzzy data. The procedures derive the degrees of acceptability of the null and the alternative hypotheses, which are explained for the case that the data are observations of a normal fuzzy random sample with unknown fuzzy mean $\tilde{\theta}$ and known variance $\sigma^{2}$, i.e. $\mathcal{X}_{1}, \ldots, \mathcal{X}_{n} \stackrel{i i d}{\sim} N\left(\tilde{\theta}, \sigma^{2}\right)$.

1. Testing $H_{0}: \tilde{\theta}=\tilde{\theta}_{0}$ versus $H_{1}: \tilde{\theta} \neq \tilde{\theta}_{0}$

Step 1. First we transform the original testing problem

$$
H_{0}: \tilde{\theta}=\tilde{\theta}_{0} \quad \text { versus } \quad H_{1}: \tilde{\theta} \neq \tilde{\theta}_{0},
$$

into a set of crisp testing problems concerning $\alpha$-levels of the fuzzy parameter. For each $\alpha$-level, based on the samples $\mathbf{X}_{\alpha}^{l}=\left(X_{1 \alpha}^{l}, \ldots, X_{n \alpha}^{l}\right)$ and $\mathbf{X}_{\alpha}^{u}=\left(X_{1 \alpha}^{u}, \ldots, X_{n \alpha}^{u}\right)$, the following classical testing problems are solved at level $\beta$

$$
\begin{array}{lll}
H_{0}: \theta_{\alpha}^{l}=\theta_{0 \alpha}^{l} & \text { versus } & H_{1}: \theta_{\alpha}^{l} \neq \theta_{0 \alpha}^{l}, \\
H_{0}: \theta_{\alpha}^{u}=\theta_{0 \alpha}^{u} & \text { versus } & H_{1}: \theta_{\alpha}^{u} \neq \theta_{0 \alpha}^{u},
\end{array}
$$

where $\tilde{\theta}_{\alpha}=\left[\theta_{\alpha}^{l}, \theta_{\alpha}^{u}\right]$ and $\tilde{\theta}_{0 \alpha}=\left[\theta_{0 \alpha}^{l}, \theta_{0 \alpha}^{u}\right]$.

Step 2. We obtain the $1-\beta$ confidence intervals for the crisp parameters $\theta_{\alpha}^{l}$ and $\theta_{\alpha}^{u}$ for each $\alpha \in(0,1]$, denoted by $\left[L_{1}\left(\mathbf{X}_{\alpha}^{l}\right), L_{2}\left(\mathbf{X}_{\alpha}^{l}\right)\right]$ and $\left[U_{1}\left(\mathbf{X}_{\alpha}^{u}\right), U_{2}\left(\mathbf{X}_{\alpha}^{u}\right)\right]$, respectively.

Step 3. We test the hypotheses (4) and (5) by investigating if the two-sided $1-\beta$ confidence intervals $\left[L_{1}\left(\mathbf{X}_{\alpha}^{l}\right), L_{2}\left(\mathbf{X}_{\alpha}^{l}\right)\right]$ and $\left[U_{1}\left(\mathbf{X}_{\alpha}^{u}\right), U_{2}\left(\mathbf{X}_{\alpha}^{u}\right)\right]$ contain $\theta_{0 \alpha}^{l}$ and $\theta_{0 \alpha}^{u}$, respectively. In fact, the test functions can be shown to be the following:

$$
\begin{aligned}
& \varphi\left(\mathbf{X}_{\alpha}^{l} ; \theta_{0 \alpha}^{l}\right)=\left\{\begin{array}{l}
0 \text { if } \theta_{0 \alpha}^{l} \in\left[L_{1}\left(\mathbf{X}_{\alpha}^{l}\right), L_{2}\left(\mathbf{X}_{\alpha}^{l}\right)\right], \\
1 \text { if } \theta_{0 \alpha}^{l} \notin\left[L_{1}\left(\mathbf{X}_{\alpha}^{l}\right), L_{2}\left(\mathbf{X}_{\alpha}^{l}\right)\right],
\end{array}\right. \\
& \varphi\left(\mathbf{X}_{\alpha}^{u} ; \theta_{0 \alpha}^{u}\right)= \begin{cases}0 & \text { if } \theta_{0 \alpha}^{u} \in\left[U_{1}\left(\mathbf{X}_{\alpha}^{u}\right), U_{2}\left(\mathbf{X}_{\alpha}^{u}\right)\right], \\
1 & \text { if } \theta_{0 \alpha}^{u} \notin\left[U_{1}\left(\mathbf{X}_{\alpha}^{u}\right), U_{2}\left(\mathbf{X}_{\alpha}^{u}\right)\right] .\end{cases}
\end{aligned}
$$

Example 2. Let $\mathcal{X}_{1}, \ldots, \mathcal{X}_{n} \stackrel{\text { iid }}{\sim} N\left(\tilde{\theta}, \sigma^{2}\right)$, with unknown fuzzy mean $\tilde{\theta}$ and known crisp variance $\sigma^{2}$. Then, we have $X_{1 \alpha}^{l}, \ldots, X_{n \alpha}^{l} \stackrel{i i d}{\sim} N\left(\theta_{\alpha}^{l}, \sigma^{2}\right)$ and $X_{1 \alpha}^{u}, \ldots, X_{n \alpha}^{u} \stackrel{i i d}{\sim} N\left(\theta_{\alpha}^{u}, \sigma^{2}\right)$. 
Therefore, the two-sided symmetric confidence intervals for parameters $\theta_{\alpha}^{l}$ and $\theta_{\alpha}^{u}$, at confidence level $1-\beta$, can easily be derived as

$$
\begin{aligned}
& S_{T}\left(\boldsymbol{X}_{\alpha}^{l}\right)=\left\{\theta_{\alpha}^{l}:\left|\frac{\sqrt{n}\left(\bar{X}_{\alpha}^{l}-\theta_{\alpha}^{l}\right)}{\sigma}\right| \leq z_{1-\frac{\beta}{2}}\right\}=\left[\bar{X}_{\alpha}^{l}-\frac{\sigma}{\sqrt{n}} z_{1-\frac{\beta}{2}}, \bar{X}_{\alpha}^{l}+\frac{\sigma}{\sqrt{n}} z_{1-\frac{\beta}{2}}\right] \\
& S_{T}\left(\boldsymbol{X}_{\alpha}^{u}\right)=\left\{\theta_{\alpha}^{u}:\left|\frac{\sqrt{n}\left(\bar{X}_{\alpha}^{u}-\theta_{\alpha}^{u}\right)}{\sigma}\right| \leq z_{1-\frac{\beta}{2}}\right\}=\left[\bar{X}_{\alpha}^{u}-\frac{\sigma}{\sqrt{n}} z_{1-\frac{\beta}{2}}, \bar{X}_{\alpha}^{u}+\frac{\sigma}{\sqrt{n}} z_{1-\frac{\beta}{2}}\right]
\end{aligned}
$$

where, $\bar{X}_{\alpha}^{j}=n^{-1} \sum_{i=1}^{n} X_{i h}^{j}$ for $j=l, u$.

So, the test functions for testing hypotheses (4) and (5) are obtained as

$$
\begin{aligned}
& \varphi\left(\boldsymbol{X}_{\alpha}^{l} ; \theta_{0 \alpha}^{l}\right)=\left\{\begin{array}{l}
0 \text { if } \theta_{0 \alpha}^{l} \in\left[\bar{X}_{\alpha}^{l}-\frac{\sigma}{\sqrt{n}} z_{1-\frac{\beta}{2}}, \bar{X}_{\alpha}^{l}+\frac{\sigma}{\sqrt{n}} z_{1-\frac{\beta}{2}}\right], \\
1 \text { if } \theta_{0 \alpha}^{l} \notin\left[\bar{X}_{\alpha}^{l}-\frac{\sigma}{\sqrt{n}} z_{1-\frac{\beta}{2}}, \bar{X}_{\alpha}^{l}+\frac{\sigma}{\sqrt{n}} z_{1-\frac{\beta}{2}}\right],
\end{array}\right. \\
& \varphi\left(\boldsymbol{X}_{\alpha}^{u} ; \theta_{0 \alpha}^{u}\right)=\left\{\begin{array}{l}
0 \text { if } \theta_{0 \alpha}^{u} \in\left[\bar{X}_{\alpha}^{u}-\frac{\sigma}{\sqrt{n}} z_{1-\frac{\beta}{2}}, \bar{X}_{\alpha}^{u}+\frac{\sigma}{\sqrt{n}} z_{1-\frac{\beta}{2}}\right] \\
1 \text { if } \theta_{0 \alpha}^{u} \notin\left[\bar{X}_{\alpha}^{u}-\frac{\sigma}{\sqrt{n}} z_{1-\frac{\beta}{2}}, \bar{X}_{\alpha}^{u}+\frac{\sigma}{\sqrt{n}} z_{1-\frac{\beta}{2}}\right] .
\end{array}\right.
\end{aligned}
$$

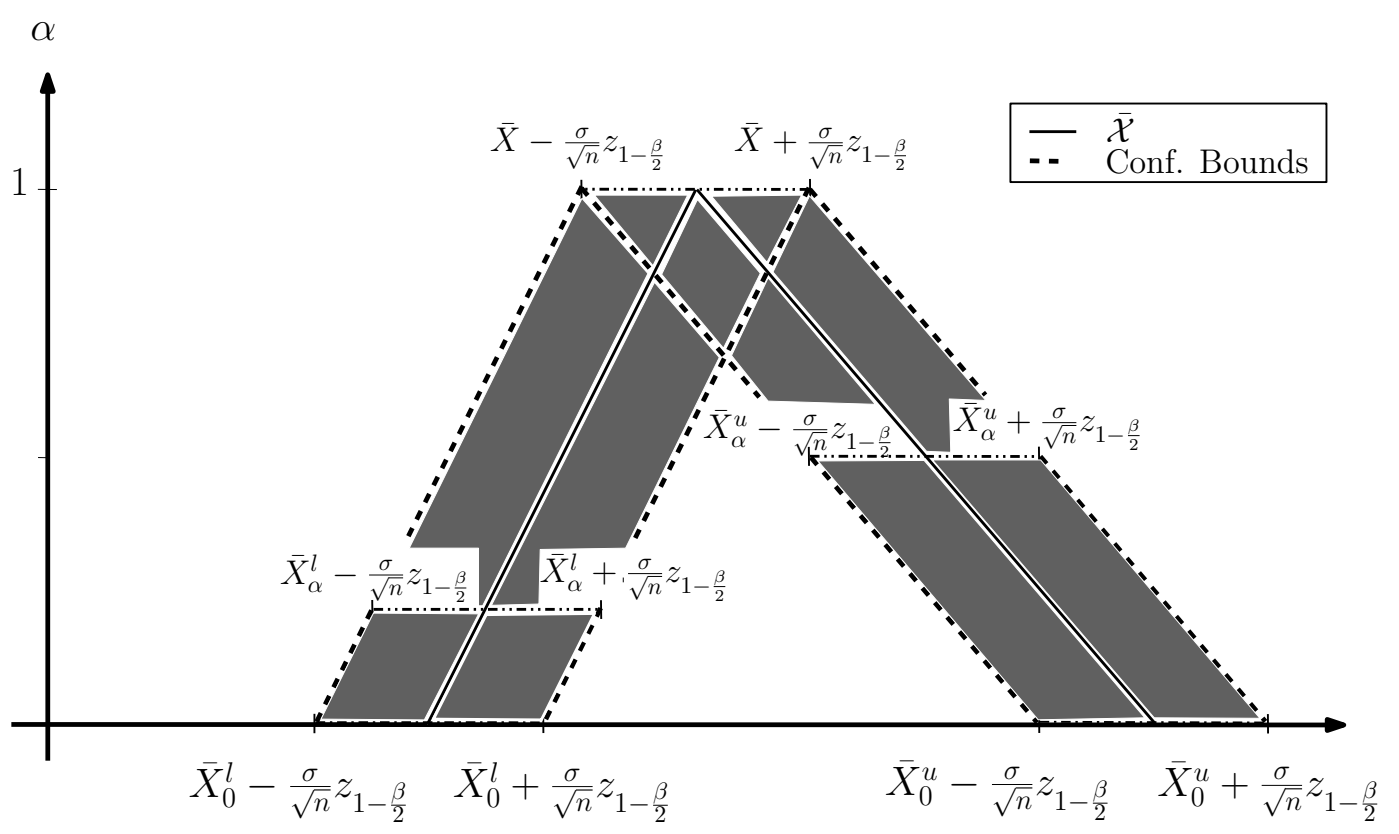

Figure 1: Graphical representation of the confidence bound constructed from the class of two-sided $(1-\beta)$ confidence intervals in Step 4.

Step 4. We aggregate the results in Step 3, in order to construct a fuzzy test. The purpose of this aggregation is to obtain a fuzzy confidence interval which helps us to derive a fuzzy test based on the degree of membership of each fuzzy parameter $\tilde{\theta}$ in the fuzzy confidence interval. To do this, first, the values of $\alpha$ for which the null hypotheses (4) and (5) are accepted or rejected, need to be categorized. In order to categorize the values of $\alpha$ easily, 


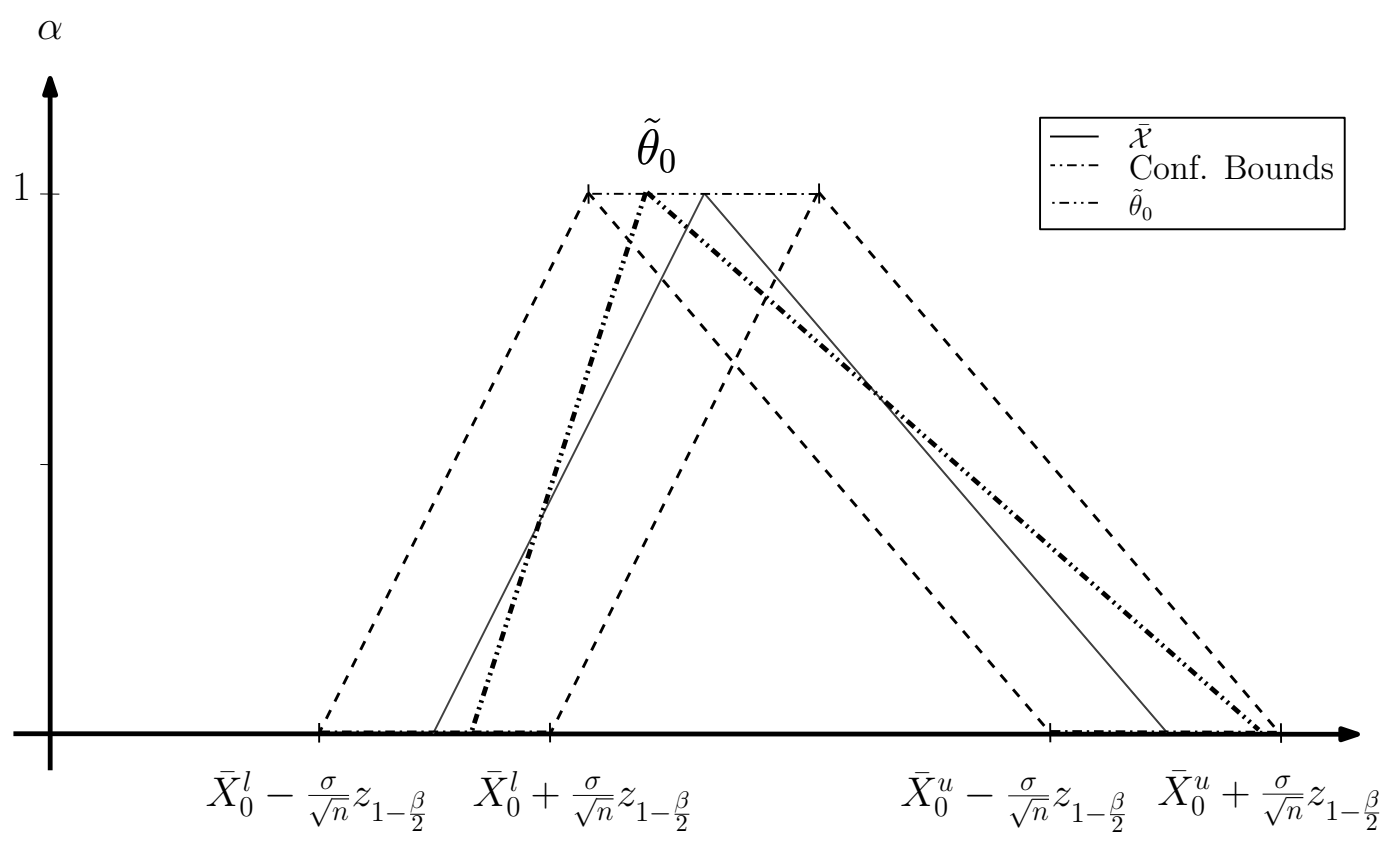

Figure 2: The hypothesis $H_{0}: \tilde{\theta}=\tilde{\theta}_{0}$ is completely accepted.

we utilize the confidence bound constructed from the confidence intervals in (6) and (7), as shown in Figure 1. To construct this plot, by beginning from 0 to 1, we put together the confidence intervals in (6) and (7) in such a way that every confidence interval receives $\alpha$ as its height. Now, by considering the membership function of the fuzzy parameter $\tilde{\theta}_{0}$ and comparing it with the confidence bound, we can determine the values of $\alpha$ for which the null hypotheses in (4) and (5) are accepted or rejected (see Figures 2, 3, and 4).

Remark 1. Suppose we want to test $H_{0}: \tilde{\theta}=\tilde{\theta}_{0}$ where $\tilde{\theta}_{0}$ is the fuzzy parameter shown in Figure 2. It is clear that for each $\alpha \in[0,1], \theta_{0 \alpha}^{l} \in S_{T}\left(\boldsymbol{X}_{\alpha}^{l}\right)$ and $\theta_{0 \alpha}^{u} \in S_{T}\left(\boldsymbol{X}_{\alpha}^{u}\right)$. In this case, since we accept the null hypotheses (4) and (5) for each $\alpha \in[0,1]$, it is reasonable that the hypothesis $H_{0}: \tilde{\theta}=\tilde{\theta}_{0}$ should completely be accepted. On the other hand, consider the case of testing $H_{0}: \tilde{\theta}=\tilde{\theta}_{0}$ where $\tilde{\theta}_{0}$ is the fuzzy parameter as shown in Figure 3. Since for each $\alpha \in[0,1], \theta_{0 \alpha}^{l} \notin S_{T}\left(\boldsymbol{X}_{\alpha}^{l}\right)$ and $\theta_{0 \alpha}^{u} \notin S_{T}\left(\boldsymbol{X}_{\alpha}^{u}\right)$, therefore, we reject the null hypotheses (4) and (5) for each $\alpha \in[0,1]$. So, it is reasonable that the hypothesis $H_{0}: \tilde{\theta}=\tilde{\theta}_{0}$ should completely be rejected.

Now, consider the problem of testing the hypothesis $H_{0}: \tilde{\theta}=\tilde{\theta}_{0}$, where $\tilde{\theta}_{0}$ is the fuzzy parameter shown in Figure 4. In this case, the relations $\theta_{0 \alpha}^{l} \in S_{T}\left(\boldsymbol{X}_{\alpha}^{l}\right)$ and $\theta_{0 \alpha}^{u} \in S_{T}\left(\boldsymbol{X}_{\alpha}^{u}\right)$ might be held for some values of $\alpha \in[0,1]$. Therefore, it is reasonable to accept the hypothesis $H_{0}: \tilde{\theta}=\tilde{\theta}_{0}$ with some degree of acceptability. To determine such a degree of acceptability, we use the concept of fuzzy confidence interval, which will be described in the next step.

Step 5. In this step, we employ the procedure introduced by (Chachi and Taheri, 2011) to construct a fuzzy confidence interval for a fuzzy parameter $\tilde{\theta}$. Upon this method, the fuzzy set $\tilde{C}_{T}=\left\{\left(\tilde{\theta}, C_{T}(\tilde{\theta})\right): \tilde{\theta} \in \mathcal{F}(\Theta)\right\}$ is obtained as a fuzzy confidence interval for the fuzzy parameter $\tilde{\theta}$. 


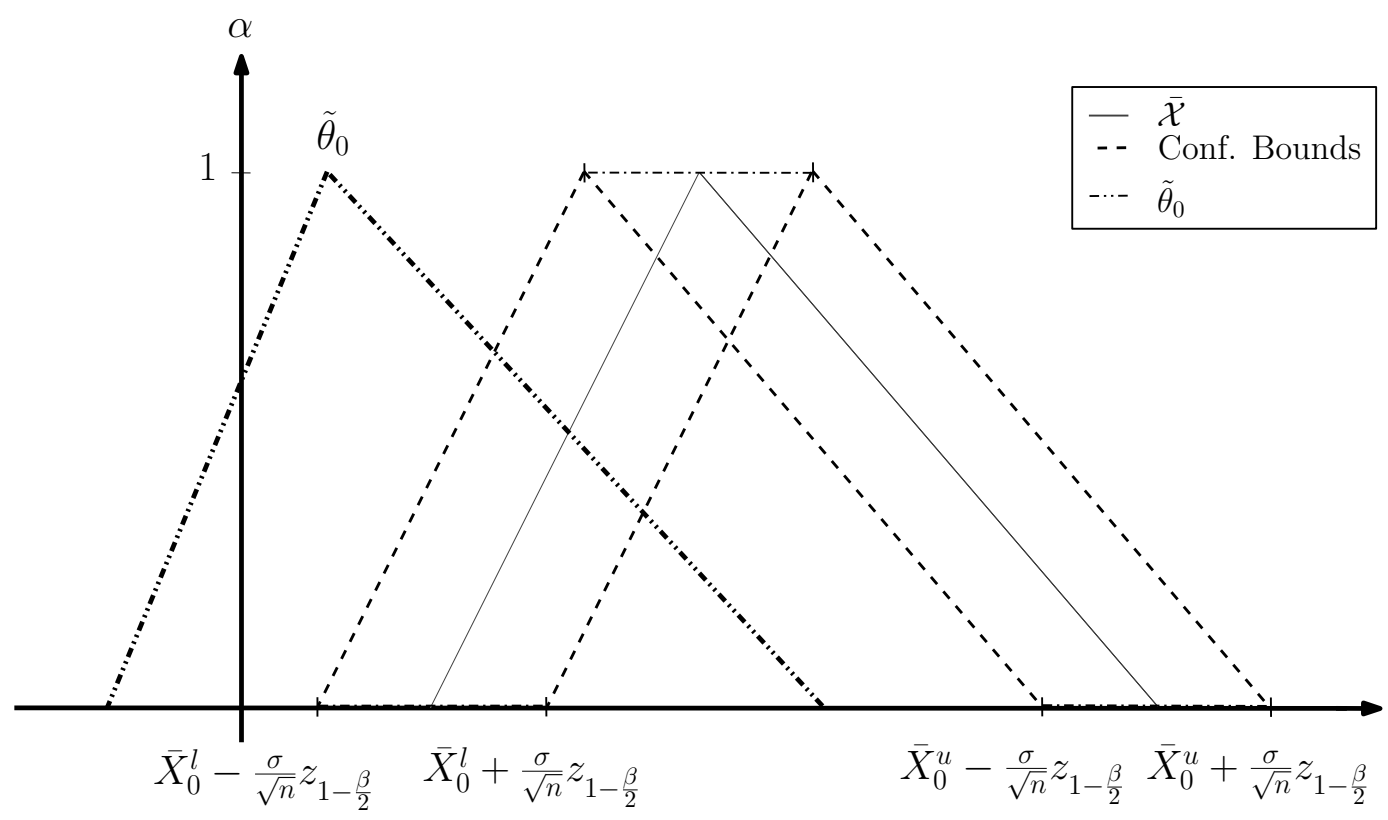

Figure 3: The hypothesis $H_{0}: \tilde{\theta}=\tilde{\theta}_{0}$ is completely rejected.

Definition 1 (Chachi and Taheri, 2011) (Re. Example 2) The degree of membership of $\tilde{\theta}$ in the two-sided fuzzy confidence interval $\tilde{C}_{T}$ is defined as $C_{T}(\tilde{\theta})=W /(W+S)$, where $W=W^{l}+W^{u}, S=S^{l}+S^{u}$, and

$$
\begin{aligned}
& K_{\tilde{\theta} ; \beta}^{l}=\left\{\alpha: \theta_{\alpha}^{l} \in\left[\bar{X}_{\alpha}^{l}-\frac{\sigma}{\sqrt{n}} z_{1-\frac{\beta}{2}}, \bar{X}_{\alpha}^{l}+\frac{\sigma}{\sqrt{n}} z_{1-\frac{\beta}{2}}\right]\right\} \text {, } \\
& C_{1 ; \tilde{\theta} ; \beta}^{l}=\left\{\alpha: \theta_{\alpha}^{l}<\bar{X}_{\alpha}^{l}-\frac{\sigma}{\sqrt{n}} z_{1-\frac{\beta}{2}}\right\}, \quad C_{2 ; \tilde{\theta} ; \beta}^{l}=\left\{\alpha: \theta_{\alpha}^{l}>\bar{X}_{\alpha}^{l}+\frac{\sigma}{\sqrt{n}} z_{1-\frac{\beta}{2}}\right\}, \\
& K_{\tilde{\theta} ; \beta}^{u}=\left\{\alpha: \theta_{\alpha}^{u} \in\left[\bar{X}_{\alpha}^{u}-\frac{\sigma}{\sqrt{n}} z_{1-\frac{\beta}{2}}, \bar{X}_{\alpha}^{u}+\frac{\sigma}{\sqrt{n}} z_{1-\frac{\beta}{2}}\right]\right\} \\
& C_{1 ; \tilde{\theta} ; \beta}^{u}=\left\{\alpha: \theta_{\alpha}^{u}<\bar{X}_{\alpha}^{u}-\frac{\sigma}{\sqrt{n}} z_{1-\frac{\beta}{2}}\right\}, \quad C_{2 ; \tilde{\theta} ; \beta}^{u}=\left\{\alpha: \theta_{\alpha}^{u}>\bar{X}_{\alpha}^{u}+\frac{\sigma}{\sqrt{n}} z_{1-\frac{\beta}{2}}\right\} \text {, } \\
& S^{l}=\int_{C_{1 ; \tilde{\theta} ; \beta}^{l}}\left[\bar{X}_{\alpha}^{l}-\frac{\sigma}{\sqrt{n}} z_{1-\frac{\beta}{2}}-\theta_{\alpha}^{l}\right] d \alpha+\int_{C_{2 ; \tilde{\theta} ; \beta}^{l}}\left[\theta_{\alpha}^{l}-\left(\bar{X}_{\alpha}^{l}+\frac{\sigma}{\sqrt{n}} z_{1-\frac{\beta}{2}}\right)\right] d \alpha, \\
& S^{u}=\int_{C_{1 ; \tilde{\theta} ; \beta}^{u}}\left[\bar{X}_{\alpha}^{u}-\frac{\sigma}{\sqrt{n}} z_{1-\frac{\beta}{2}}-\theta_{\alpha}^{u}\right] d \alpha+\int_{C_{2 ; \tilde{\theta} ; \beta}^{u}}\left[\theta_{\alpha}^{u}-\left(\bar{X}_{\alpha}^{u}+\frac{\sigma}{\sqrt{n}} z_{1-\frac{\beta}{2}}\right)\right] d \alpha, \\
& W^{l}=\int_{K_{\tilde{\theta} ; \beta}^{l}}\left[\theta_{\alpha}^{l}-\left(\bar{X}_{\alpha}^{l}-\frac{\sigma}{\sqrt{n}} z_{1-\frac{\beta}{2}}\right)\right] d \alpha+\int_{K_{\tilde{\theta} ; \beta}^{l}}\left[\bar{X}_{\alpha}^{l}+\frac{\sigma}{\sqrt{n}} z_{1-\frac{\beta}{2}}-\theta_{\alpha}^{l}\right] d \alpha \\
& =\frac{2 \sigma}{\sqrt{n}} z_{1-\frac{\beta}{2}} \mathcal{L}\left(K_{\tilde{\theta} ; \beta}^{l}\right)
\end{aligned}
$$




$$
\begin{aligned}
W^{u} & =\int_{K_{\tilde{\theta} ; \beta}^{u}}\left[\theta_{\alpha}^{u}-\left(\bar{X}_{\alpha}^{u}-\frac{\sigma}{\sqrt{n}} z_{1-\frac{\beta}{2}}\right)\right] d \alpha+\int_{K_{\tilde{\theta} ; \beta}^{u}}\left[\bar{X}_{\alpha}^{u}+\frac{\sigma}{\sqrt{n}} z_{1-\frac{\beta}{2}}-\theta_{\alpha}^{u}\right] d \alpha \\
& =\frac{2 \sigma}{\sqrt{n}} z_{1-\frac{\beta}{2}} \mathcal{L}\left(K_{\tilde{\theta} ; \beta}^{u}\right),
\end{aligned}
$$

where, $\mathcal{L}(A)$ is the Lebesgue measure of a set $A$ (see Figure 4 for some details).

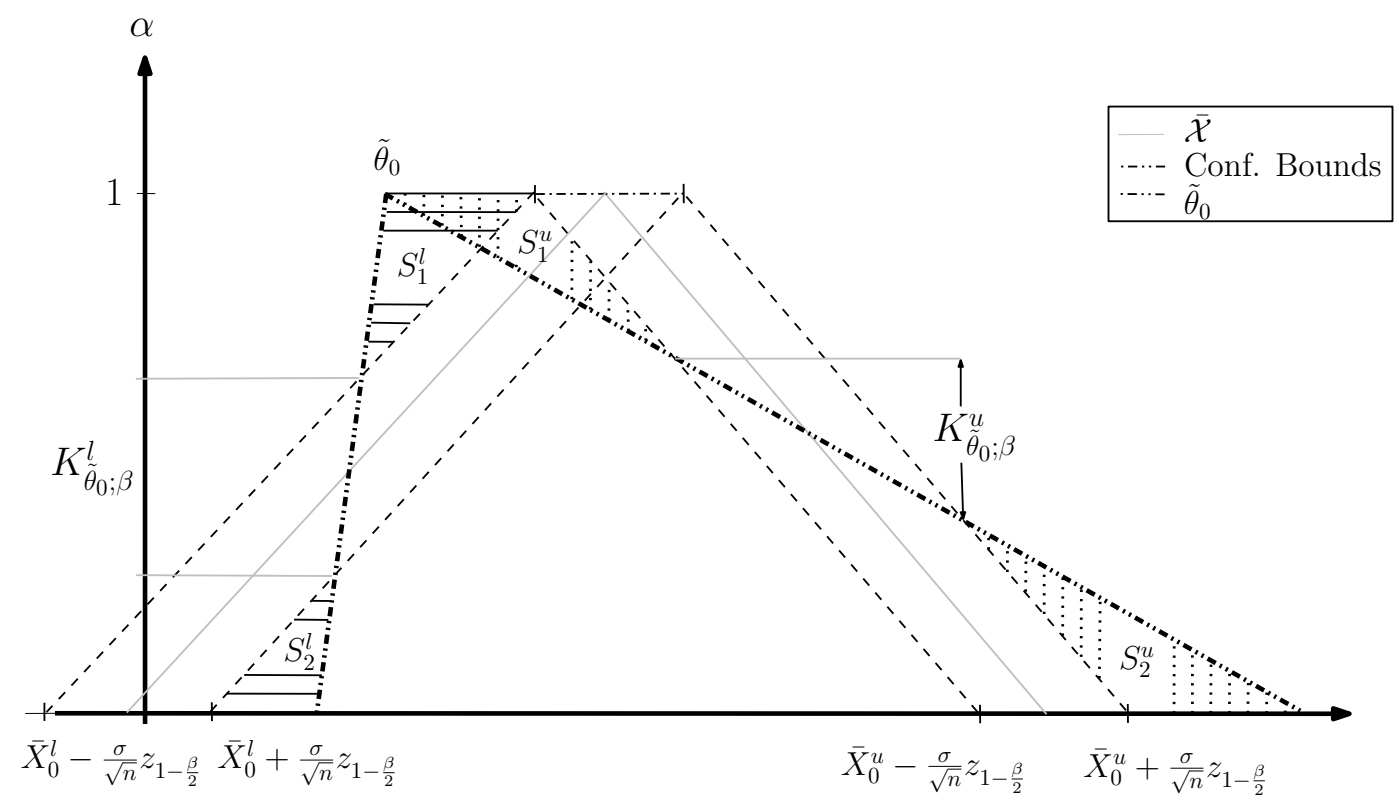

Figure 4: The hypothesis $H_{0}: \tilde{\theta}=\tilde{\theta}_{0}$ is partially accepted.

Step 6. Finally, using the result in Step 5, the following fuzzy test function is provided for testing the hypothesis $H_{0}: \tilde{\theta}=\tilde{\theta}_{0}$ versus $H_{1}: \tilde{\theta} \neq \tilde{\theta}_{0}$ at level $\beta$, based on the fuzzy random sample $\mathcal{X}_{1}, \ldots, \mathcal{X}_{n}$

$$
\tilde{\varphi}\left(\underline{\mathcal{X}} ; \tilde{\theta}_{0}\right)(t)= \begin{cases}C_{T}\left(\tilde{\theta}_{0}\right) & t=0, \\ 1-C_{T}\left(\tilde{\theta}_{0}\right) & t=1 .\end{cases}
$$

Note that the fuzzy test function $\tilde{\varphi}\left(\underline{\mathcal{X}} ; \tilde{\theta}_{0}\right): \mathcal{F}^{n}(\mathbb{R}) \rightarrow \mathcal{F}\{0,1\}$ is characterized by a fuzzy set, which accepts the null hypothesis with degree of $C_{T}\left(\tilde{\theta}_{0}\right)$ and rejects it with degree of $1-C_{T}\left(\tilde{\theta}_{0}\right)$.

Remark 2. In the above fuzzy test function $\tilde{\varphi}\left(\underline{\mathcal{X}} ; \tilde{\theta}_{0}\right)$, the values of $C_{T}\left(\tilde{\theta}_{0}\right)$ and $1-C_{T}\left(\tilde{\theta}_{0}\right)$ correspond to the degrees of acceptability of the null and alternative hypotheses, respectively, which can be interpreted as the degrees of conviction that we should accept or reject the null hypothesis. Such a fuzzy test function, contrary to the classical crisp test, does not lead to a binary decision, i.e. to accept or to reject the null hypothesis, but to a fuzzy decision. Thus, in the situation when $C_{T}\left(\tilde{\theta}_{0}\right)$ is neither 0 nor 1 , a user has to decide whether to reject or to accept the given hypothesis actually, however the value $C_{T}\left(\tilde{\theta}_{0}\right)$ would support the decision, i.e. for greater values of $C_{T}\left(\tilde{\theta}_{0}\right)$ he/she is more convinced to accept the null hypothesis. 
2. Testing $H_{0}: \tilde{\theta}=\tilde{\theta}_{0}$ versus $H_{1}: \tilde{\theta} \succ \tilde{\theta}_{0}$

Concerning the above procedure, the proposed fuzzy test for testing these hypotheses is obtained as follows

$$
\tilde{\varphi}\left(\underline{\mathcal{X}} ; \tilde{\theta}_{0}\right)(t)= \begin{cases}C_{R}\left(\tilde{\theta}_{0}\right) & t=0, \\ 1-C_{R}\left(\tilde{\theta}_{0}\right) & t=1,\end{cases}
$$

where $C_{R}\left(\tilde{\theta}_{0}\right)=W /(W+S)$ is the degree of membership of $\tilde{\theta}_{0}$ in the right one-sided fuzzy confidence interval given as

$$
\begin{aligned}
K_{\tilde{\theta} ; \beta}^{l} & =\left\{\alpha \in[0,1]: \theta_{\alpha}^{l} \in\left[\bar{X}_{\alpha}^{l}-\frac{\sigma}{\sqrt{n}} z_{1-\beta}, \infty\right)\right\} \\
C_{\tilde{\theta} ; \beta}^{l} & =\left\{\alpha \in[0,1]: \theta_{\alpha}^{l} \notin\left[\bar{X}_{\alpha}^{l}-\frac{\sigma}{\sqrt{n}} z_{1-\beta}, \infty\right)\right\}=\left(K_{\tilde{\theta} ; \beta}^{l}\right)^{c}, \\
K_{\tilde{\theta} ; \beta}^{u} & =\left\{\alpha \in[0,1]: \theta_{\alpha}^{u} \in\left[\bar{X}_{\alpha}^{u}-\frac{\sigma}{\sqrt{n}} z_{1-\beta}, \infty\right)\right\} \\
C_{\tilde{\theta} ; \beta}^{u} & =\left\{\alpha \in[0,1]: \theta_{\alpha}^{u} \notin\left[\bar{X}_{\alpha}^{u}-\frac{\sigma}{\sqrt{n}} z_{1-\beta}, \infty\right)\right\}=\left(K_{\tilde{\theta} ; \beta}^{u}\right)^{c}, \\
S & =\int_{C_{\tilde{\theta} ; \beta}^{l}}\left[\bar{X}_{\alpha}^{l}-\frac{\sigma}{\sqrt{n}} z_{1-\beta}-\theta_{\alpha}^{l}\right] d \alpha+\int_{C_{\tilde{\theta} ; \beta}^{u}}\left[\bar{X}_{\alpha}^{u}-\frac{\sigma}{\sqrt{n}} z_{1-\beta}-\theta_{\alpha}^{u}\right] d \alpha, \\
W & =\int_{K_{\tilde{\theta} ; \beta}^{l}}\left[\theta_{\alpha}^{l}-\left(\bar{X}_{\alpha}^{l}-\frac{\sigma}{\sqrt{n}} z_{1-\beta}\right)\right] d \alpha+\int_{K_{\tilde{\tilde{\theta}} ; \beta}^{u}}\left[\theta_{\alpha}^{u}-\left(\bar{X}_{\alpha}^{u}-\frac{\sigma}{\sqrt{n}} z_{1-\beta}\right)\right] d \alpha .
\end{aligned}
$$

3. Testing $H_{0}: \tilde{\theta}=\tilde{\theta}_{0}$ versus $H_{1}: \tilde{\theta} \prec \tilde{\theta}_{0}$

Similarly, the proposed fuzzy test for testing these hypotheses is obtained as

$$
\tilde{\varphi}\left(\underline{\mathcal{X}} ; \tilde{\theta}_{0}\right)(t)= \begin{cases}C_{L}\left(\tilde{\theta}_{0}\right) & t=0, \\ 1-C_{L}\left(\tilde{\theta}_{0}\right) & t=1,\end{cases}
$$

where $C_{L}\left(\tilde{\theta}_{0}\right)=W /(W+S)$ is the degree of membership of $\tilde{\theta}_{0}$ in the left one-sided fuzzy confidence interval given as

$$
\begin{aligned}
K_{\tilde{\theta} ; \beta}^{l} & =\left\{\alpha \in[0,1]: \theta_{\alpha}^{l} \in\left(-\infty, \bar{X}_{\alpha}^{l}+\frac{\sigma}{\sqrt{n}} z_{1-\beta}\right]\right\} \\
K_{\tilde{\theta} ; \beta}^{u} & =\left\{\alpha \in[0,1]: \theta_{\alpha}^{u} \in\left(-\infty, \bar{X}_{\alpha}^{u}+\frac{\sigma}{\sqrt{n}} z_{1-\beta}\right]\right\} \\
S & =\int_{C_{\tilde{\theta} ; \beta}^{l}}\left[\theta_{\alpha}^{l}-\bar{X}_{\alpha}^{l}-\frac{\sigma}{\sqrt{n}} z_{1-\beta}\right] d \alpha+\int_{C_{\tilde{\theta} ; \beta}^{u}}\left[\theta_{\alpha}^{u}-\bar{X}_{\alpha}^{u}-\frac{\sigma}{\sqrt{n}} z_{1-\beta}\right] d \alpha, \\
W & =\int_{K_{\tilde{\theta} ; \beta}^{l}}\left[\bar{X}_{\alpha}^{l}+\frac{\sigma}{\sqrt{n}} z_{1-\beta}-\theta_{\alpha}^{l}\right] d \alpha+\int_{K_{\tilde{\theta} ; \beta}^{u}}\left[\bar{X}_{\alpha}^{u}+\frac{\sigma}{\sqrt{n}} z_{1-\beta}-\theta_{\alpha}^{u}\right] d \alpha,
\end{aligned}
$$

and $C_{\tilde{\theta} ; \beta}^{l}=\left(K_{\tilde{\theta} ; \beta}^{l}\right)^{c}, C_{\tilde{\theta} ; \beta}^{u}=\left(K_{\tilde{\theta} ; \beta}^{u}\right)^{c}$. 
Table 1: Triangular fuzzy numbers as the observations of a normal FRV in Example 3.

\begin{tabular}{|c|c|c|c|c|c|c|c|}
\hline$i$ & $\mathcal{X}_{i}$ & $i$ & $\mathcal{X}_{i}$ & $i$ & $\mathcal{X}_{i}$ & $i$ & $\mathcal{X}_{i}$ \\
\hline 1 & $(45,5,6)_{T}$ & 10 & $(51,5,5)_{T}$ & 19 & $(64,7,5)_{T}$ & 28 & $(52,3,6)_{T}$ \\
2 & $(48,6,5)_{T}$ & 11 & $(49,8,5)_{T}$ & 20 & $(62,8,5)_{T}$ & 29 & $(53,5,5)_{T}$ \\
3 & $(41,3,6)_{T}$ & 12 & $(42,5,6)_{T}$ & 21 & $(59,6,5)_{T}$ & 30 & $(47,6,6)_{T}$ \\
4 & $(49,7,7)_{T}$ & 13 & $(55,8,4)_{T}$ & 22 & $(57,6,4)_{T}$ & 31 & $(41,4,7)_{T}$ \\
5 & $(39,5,5)_{T}$ & 14 & $(44,6,3)_{T}$ & 23 & $(46,6,5)_{T}$ & 32 & $(57,5,5)_{T}$ \\
6 & $(56,6,7)_{T}$ & 15 & $(61,7,5)_{T}$ & 24 & $(64,8,5)_{T}$ & 33 & $(51,5,6)_{T}$ \\
7 & $(47,6,7)_{T}$ & 16 & $(49,6,5)_{T}$ & 25 & $(57,5,5)_{T}$ & 34 & $(48,6,5)_{T}$ \\
8 & $(61,5,6)_{T}$ & 17 & $(59,5,6)_{T}$ & 26 & $(60,7,6)_{T}$ & 35 & $(46,6,8)_{T}$ \\
9 & $(43,7,7)_{T}$ & 18 & $(40,5,8)_{T}$ & 27 & $(41,5,6)_{T}$ & 36 & $(58,6,5)_{T}$ \\
\hline
\end{tabular}

\section{Numerical Examples}

In this section, in order to explain the applicability of the proposed approach, some numerical examples are provided.

Example 3 (Wu, 2009). Suppose that the triangular fuzzy data in Table 1 are the observations of a sample of 36 from a normal $F R V$ with unknown fuzzy mean $\tilde{\theta} \in \mathcal{F}(\mathbb{R})$ and known $\sigma^{2}=64$. Assume that we wish to test the hypothesis

$$
H_{0}: \tilde{\theta}=(48,5,6)_{T} \text { versus } H_{1}: \tilde{\theta} \neq(48,5,6)_{T},
$$

at level $\beta=0.10$.

According to the procedure described in Section 4, we have

$$
\begin{aligned}
\overline{\mathcal{X}} & =(51.17,5.81,5.61)_{T}, \\
\overline{\mathcal{X}}_{\alpha} & =\left[\bar{x}_{\alpha}^{l}, \bar{x}_{\alpha}^{u}\right]=[45.36+5.81 \alpha, 56.78-5.61 \alpha], \\
\tilde{\theta}_{0 \alpha} & =\left[\theta_{0 \alpha}^{l}, \theta_{0 \alpha}^{u}\right]=[43+5 \alpha, 54-6 \alpha] .
\end{aligned}
$$

Now, based on the samples $\boldsymbol{x}_{\alpha}^{l}=\left(x_{1 \alpha}^{l}, \ldots, x_{n \alpha}^{l}\right)$ and $\boldsymbol{x}_{\alpha}^{u}=\left(x_{1 \alpha}^{u}, \ldots, x_{n \alpha}^{u}\right)$, we test the following hypotheses

$$
\begin{array}{lll}
H_{0}: \theta_{\alpha}^{l}=43+5 \alpha & \text { versus } & H_{1}: \theta_{\alpha}^{l} \neq 43+5 \alpha, \\
H_{0}: \theta_{\alpha}^{u}=54-6 \alpha \quad \text { versus } & H_{1}: \theta_{\alpha}^{u} \neq 54-6 \alpha,
\end{array}
$$

at level $\beta=0.10$, by using the corresponding two-sided confidence intervals at confidence level $1-\beta=0.90$. The usual confidence intervals for the parameters $\theta_{\alpha}^{l}$ and $\theta_{\alpha}^{u}$ are easily obtained as

$$
\begin{aligned}
& S_{T}\left(\boldsymbol{x}_{\alpha}^{l}\right)=[43.17+5.81 \alpha, 47.55+5.81 \alpha], \\
& S_{T}\left(\boldsymbol{x}_{\alpha}^{u}\right)=[54.59-5.61 \alpha, 58.97-5.61 \alpha] .
\end{aligned}
$$


Table 2: Data set in Example 4.

\begin{tabular}{|c|c|c|c|c|c|c|c|c|c|c|c|}
\hline$i$ & $x_{i}$ & $i$ & $x_{i}$ & $i$ & $x_{i}$ & $i$ & $x_{i}$ & $i$ & $x_{i}$ & $i$ & $x_{i}$ \\
\hline 1 & 38.0 & 5 & 35.0 & 9 & 45.0 & 13 & 60.0 & 17 & 49.0 & 21 & 52.0 \\
2 & 41.0 & 6 & 43.0 & 10 & 50.0 & 14 & 52.0 & 18 & 44.0 & 22 & 42.0 \\
3 & 47.5 & 7 & 54.0 & 11 & 58.6 & 15 & 52.0 & 19 & 49.0 & 23 & 40.0 \\
4 & 51.0 & 8 & 52.0 & 12 & 62.0 & 16 & 49.0 & 20 & 50.3 & 24 & 37.0 \\
& & & & & & & & & & 25 & 21.2 \\
\hline
\end{tabular}

Based on the above confidence intervals, the following test functions are obtained for testing the hypotheses (8) and (9)

$$
\begin{aligned}
& \varphi\left(\boldsymbol{x}_{\alpha}^{l} ; \theta_{0 \alpha}^{l}\right)= \begin{cases}0 & \text { if } \theta_{0 \alpha}^{l} \in[43.17+5.81 \alpha, 47.55+5.81 \alpha], \\
1 & \text { if } \theta_{0 \alpha}^{l} \notin[43.17+5.81 \alpha, 47.55+5.81 \alpha],\end{cases} \\
& \varphi\left(\boldsymbol{x}_{\alpha}^{u} ; \theta_{0 \alpha}^{u}\right)= \begin{cases}0 & \text { if } \theta_{0 \alpha}^{u} \in[54.59-5.61 \alpha, 58.97-5.61 \alpha], \\
1 & \text { if } \theta_{0 \alpha}^{u} \notin[54.59-5.61 \alpha, 58.97-5.61 \alpha] .\end{cases}
\end{aligned}
$$

The results of the above test functions lead to reject the null hypotheses in (8) and (9), for each $\alpha \in[0,1]$. On the other hand, the following sets are easily obtained

$$
\begin{array}{rlrl}
K_{\tilde{\theta}_{0} ; 0.10}^{l} & =\emptyset, & C_{1 ; \tilde{\theta}_{0} ; 0.10}^{l}=[0,1], & C_{2 ; \tilde{\theta}_{0} ; 0.10}^{l}=\emptyset, \\
K_{\tilde{\theta}_{0} ; 0.10}^{u} & =\emptyset, \quad C_{1 ; \tilde{\theta}_{0} ; 0.10}^{u}=[0,1], & C_{2 ; \tilde{\theta}_{0} ; 0.10}^{u}=\emptyset, \\
S^{l} & =0.575, \quad S^{u}=0.785, & S=1.360, \\
W^{l}=0, & W^{u}=0, \quad W=0 .
\end{array}
$$

So, based on the fuzzy observations and at level $1-\beta=0.90$, the fuzzy parameter $\tilde{\theta}_{0}=$ $(48,5,6)_{T}$ has no degree of membership in the two-sided fuzzy confidence interval, i.e. $C_{T}\left(\tilde{\theta}_{0}\right)=0$. Therefore, the hypothesis $H_{0}: \tilde{\theta}=(48,5,6)_{T}$ versus $H_{1}: \tilde{\theta} \neq(48,5,6)_{T}$ is accepted with degree of acceptability $C_{T}\left(\tilde{\theta}_{0}\right)=0$, and the fuzzy test function is obtained as

$$
\tilde{\varphi}\left(\underline{\mathcal{X}} ; \tilde{\theta}_{0}\right)(t)= \begin{cases}0 & t=0 \\ 1 & t=1\end{cases}
$$

Example 4. One of the classical problems in soil science is the measurement of some physical, chemical and/or biological soil properties. The problem results from the difficulty and time of measurements which yield imprecise observations. In studying natural systems such as soil systems, in which we come across imprecise observations, we must therefore attempt to analyze the behavior of such systems more realistically.

To analyze some characteristics of the soil, especially the SP (which shows soil saturated by water), a study has been done in a part of Silakhor plain situated in the province of Lorestan (west of Iran), between the cities of Broujerd and Durood. The 100 hectares field, located in the middle of the plain, were sampled on a grid with intersections at 
$200(\mathrm{~m})$ interval. A total of 25 core samples were obtained from $0.0(\mathrm{~cm})$ to $25(\mathrm{~cm})$ depth. The SP were measured using standard procedures. The data set is given in Table 2 (Mohammadi and Taheri, 2004). We can assume that, based on a pilot study, such data are distributed according to a normal distribution with $\sigma=9$.

However, due to some difficulties in measurements, we are not sure about the crispness of the observations. So, it is convenient to consider the data as fuzzy observations. To convert the data set in Table 2 to a set of fuzzy numbers, we may employ a fuzzy representation method. There are several families of fuzzy representations in the literature (González-Rodríguez, Colubi, and Gil, 2006a; Colubi, González-Rodríguez, Lubiano, and Montenegro, 2006; Colubi and González-Rodríguez, 2007). A family of interesting fuzzy representations is proposed in González-Rodríguez et al. (2006a). Each representation transforms crisp data (the variable values of a real-valued random variable) into fuzzy sets (the associated FRV values) by mapping $\tilde{\gamma}: \mathbb{R} \rightarrow \mathcal{F}(\mathbb{R})$ whose membership function and $\alpha$-level sets are given by

$$
\begin{aligned}
& \tilde{\gamma}_{x}(t)= \begin{cases}1-(x-t)^{h_{L}(x)} & \text { if } t \in[x-1, x], \\
1-(t-x)^{h_{R}(x)} & \text { if } t \in[x, x+1], \\
0 & \text { otherwise, }\end{cases} \\
& \left(\tilde{\gamma}_{x}\right)_{\alpha}=\left[x-(1-\alpha)^{1 / h_{L}(x)}, x+(1-\alpha)^{1 / h_{R}(x)}\right] \quad \forall \alpha \in(0,1],
\end{aligned}
$$

where

$$
\begin{aligned}
& h_{L}(x)= \begin{cases}\frac{1}{1+\frac{x-x_{0}}{a}} \quad \text { if } x \geq x_{0}, \\
1-\frac{x-x_{0}}{a} \quad \text { if } x<x_{0},\end{cases} \\
& h_{R}(x)=\frac{1}{h_{L}(x)} \quad \forall x \in \mathbb{R},
\end{aligned}
$$

for $x_{0} \in \mathbb{R}$ and $a \in \mathbb{R}^{+}$. The $\mathcal{F}(\mathbb{R})$-valued $F R V \tilde{\gamma}_{X}: \Omega \rightarrow \mathcal{F}(\mathbb{R})$ will be called the $\gamma$-fuzzy representation of $X$.

The membership functions of fuzzy representations for some observations are given in Figure 5 for the values 38, 41, 47.5, and 51 (the first four data), when $x_{0}=46.98$ and $a=1$. The $\alpha$-level set of the fuzzy sample mean is obtained as

$$
\overline{\mathcal{X}}_{\alpha}=\left[46.98-\frac{1}{25} \sum_{i=1}^{25}(1-\alpha)^{1 / h_{L}\left(x_{i}\right)}, 46.98+\frac{1}{25} \sum_{i=1}^{25}(1-\alpha)^{1 / h_{R}\left(x_{i}\right)}\right] \quad \forall \alpha \in(0,1] .
$$

Suppose that, based on the above soil fuzzy data, we want to test the hypothesis $H_{0}: \tilde{\theta}=$ $\tilde{\gamma}_{44.25}$ versus $H_{1}: \tilde{\theta} \prec \tilde{\gamma}_{44.25}$ at level $\beta=0.05$. Note that, $\tilde{\gamma}_{44.25}$ is the fuzzy perception of $\theta_{0}=44.25$. The membership function and the $\alpha$-level set of $\tilde{\gamma}_{44.25}$ are given as

$$
\begin{aligned}
& \tilde{\gamma}_{\theta_{0}}(t)= \begin{cases}1-\left(\theta_{0}-t\right)^{h_{L}\left(\theta_{0}\right)} & \text { if } t \in\left[\theta_{0}-1, \theta_{0}\right], \\
1-\left(t-\theta_{0}\right)^{h_{R}\left(\theta_{0}\right)} & \text { if } t \in\left[\theta_{0}, \theta_{0}+1\right], \\
0 & \text { otherwise, }\end{cases} \\
& \left(\tilde{\gamma}_{\theta_{0}}\right)_{\alpha}=\left[\left(\gamma_{\theta_{0}}\right)_{\alpha}^{l},\left(\gamma_{\theta_{0}}\right)_{\alpha}^{u}\right]=\left[\theta_{0}-(1-\alpha)^{1 / h_{L}\left(\theta_{0}\right)}, \theta_{0}+(1-\alpha)^{1 / h_{R}\left(\theta_{0}\right)}\right] \quad \forall \alpha \in(0,1] .
\end{aligned}
$$




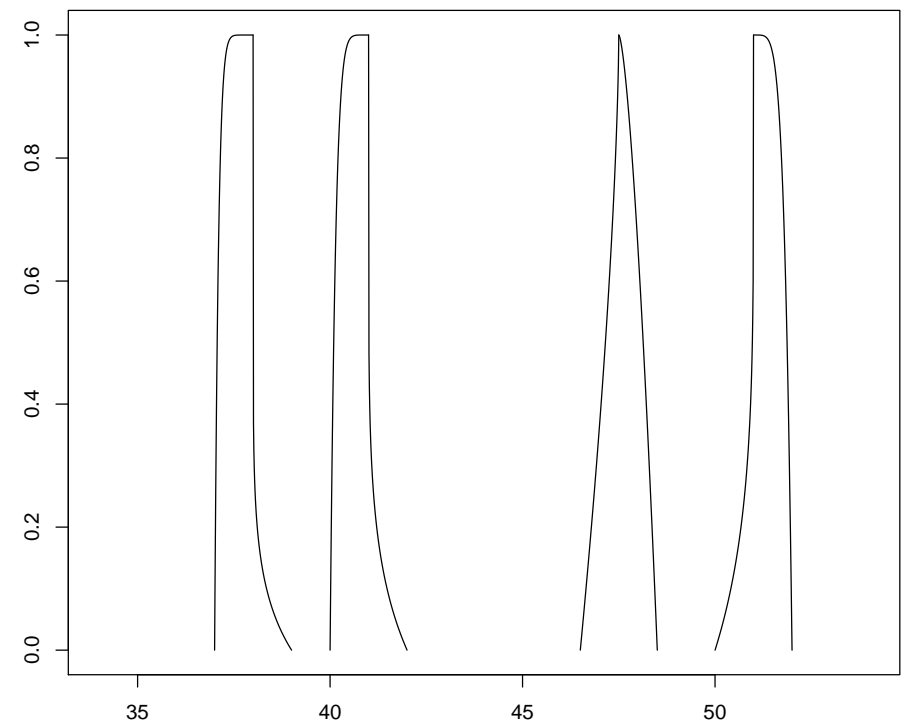

Figure 5: The fuzzy values $\tilde{\gamma}_{x_{i}}$ for $i=1,2,3,4, x_{0}=46.98$ and $a=1$ in Example 4.

Figure 6 shows the mean value of the $\gamma$-fuzzy representation of the random sample, i.e. $\overline{\mathcal{X}}$, and the fuzzy parameter $\tilde{\gamma}_{\theta_{0}}$.

Based on the procedure proposed in Section 4, the right one-sided 0.95 confidence intervals for the parameters $\left(\gamma_{\theta_{0}}\right)_{\alpha}^{l}$ and $\left(\gamma_{\theta_{0}}\right)_{\alpha}^{u}$ are obtained as

$$
\begin{aligned}
& S_{R}\left(\boldsymbol{x}_{\alpha}^{l}\right)=\left[46.98-\frac{1}{25} \sum_{i=1}^{25}(1-\alpha)^{1 / h_{L}\left(x_{i}\right)}-\frac{9}{5} 1.645, \infty\right), \\
& S_{R}\left(\boldsymbol{x}_{\alpha}^{u}\right)=\left[46.98+\frac{1}{25} \sum_{i=1}^{25}(1-\alpha)^{1 / h_{R}\left(x_{i}\right)}-\frac{9}{5} 1.645, \infty\right) .
\end{aligned}
$$

In addition, the following results are easily obtained by comparing the above confidence intervals and the $\alpha$-level sets of $\tilde{\gamma}_{\theta_{0}}$

$$
\begin{aligned}
& K_{\tilde{\gamma}_{0} ; 0.05}^{l}=[0,0.092) \cup(0.921,1], \quad C_{\tilde{\gamma}_{\theta_{0}} ; 0.05}^{l}=[0.092,0.921], \quad S=0.2542, \\
& K_{\tilde{\gamma}_{0} ; 0.05}^{u}=[0,0.212) \cup(0.994,1], \quad C_{\tilde{\gamma}_{\theta_{0}} ; 0.05}^{u}=[0.212,0.994], \quad W=0.0426 .
\end{aligned}
$$

The degree of membership of $\tilde{\gamma}_{\theta_{0}}$ in the right one-sided fuzzy confidence interval is obtained as $C_{R}\left(\tilde{\gamma}_{\theta_{0}}\right)=0.0426 /(0.0426+0.2542)=0.1435$. Finally, we have the following test function

$$
\tilde{\varphi}\left(\underline{\mathcal{X}} ; \tilde{\gamma}_{\theta_{0}}\right)(t)= \begin{cases}0.1435 & \text { if } t=0 \\ 0.8565 & \text { if } t=1\end{cases}
$$

With other words, based on the fuzzy observations and at level 0.95 , the amount of possibility that the true mean of population be $\tilde{\gamma}_{\theta_{0}}$, is 0.1435 . Therefore, at level $\beta=0.05$, the 


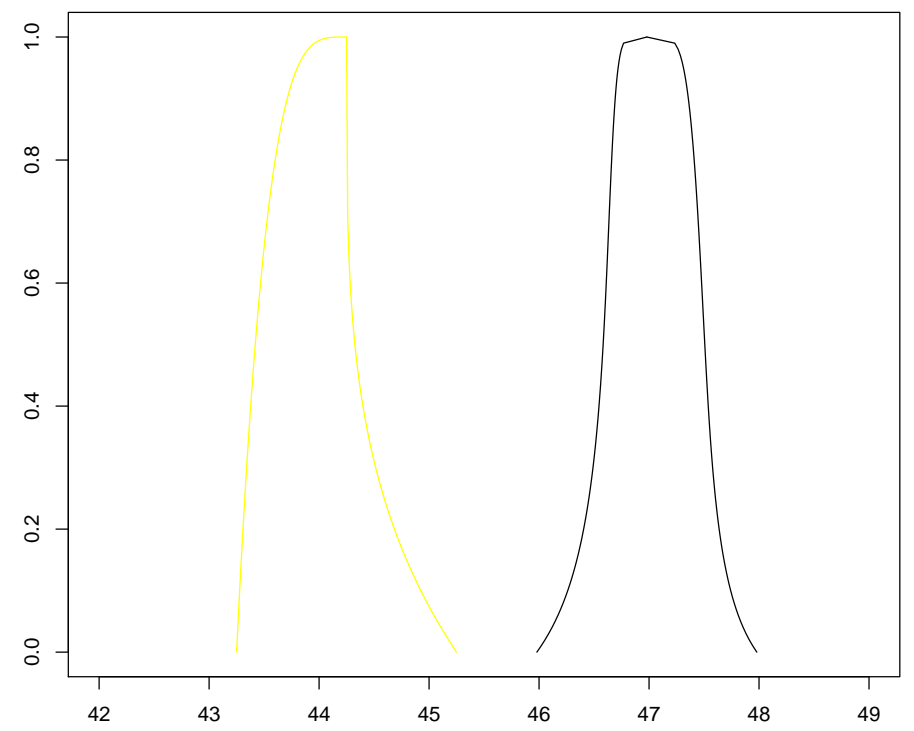

Figure 6: The mean value of the $\gamma$-fuzzy representation of the random variable and the null hypothesized value when $x_{0}=46.98$ and $a=1$.

hypothesis $H_{0}: \tilde{\theta}=\tilde{\gamma}_{\theta_{0}}$ is accepted against the hypothesis $H_{1}: \tilde{\theta} \succ \tilde{\gamma}_{\theta_{0}}$ with degree of acceptability $C_{R}\left(\tilde{\gamma}_{\theta_{0}}\right)=0.1435$.

\section{Conclusion}

In the present work, based on the concept of fuzzy confidence interval, we introduced the so called fuzzy test for testing statistical hypotheses about an imprecise parameter when the data are reported as fuzzy numbers. In the proposed approach, the available data are assumed to be the observations of FRVs.

A well-known method of constructing fuzzy confidence intervals is used to determine the degree of membership of each fuzzy parameter in the fuzzy confidence interval and then to make inference in testing a hypothesis about the fuzzy parameter of interest. The proposed fuzzy test, contrary to the classical crisp test, does not lead to a binary decision, i.e. to accept or to reject the null hypothesis, but to a fuzzy decision. Based on the proposed approach, the decision makers actually accept or reject the given hypothesis about a fuzzy parameter, however an index, called the degree of acceptability, would support the decision. Such a fuzzy test is a natural generalization of the traditional statistical tests, i.e. if the data and the parameter(s) of interest are precise, we get classical statistical tests with the binary decision as a special case.

Developing the suggested procedure to other FRVs, rather than normal, and to a nonparametric context are interesting subjects for future work. Moreover, a study of properties of the proposed procedure, and the effect of sample size in the obtained fuzzy test are 
other possible lines for future work.

\section{References}

Akbari, M. G., and Rezaei, A. (2009). Bootstrap statistical inference for the variance based on fuzzy data. Austrian Journal of Statistics, 121-130.

Arefi, M., and Taheri, S. M. (2011). Testing fuzzy hypotheses using fuzzy data based on fuzzy test statistic. Journal of Uncertain Systems, 5, 45-61.

Arnold, B. F., and Gerke, O. (2003). Testing fuzzy hypotheses in linear regression models. Metrika, 57, 81-95.

Aumann, R. J. (1965). Integrals of set-valued functions. Journal of Mathematical Analysis and Applications, 12, 1-12.

Beaulieu-Préost, D. (2006). Confidence intervals: From test of statistical significance to confidence intervals, range hypotheses and substantial effects. Tutorials in Quantitative Methods for Psychology, 2, 11-19.

Bolviken, E., and Skovlund, E. (1996). Confidence intervals from Monte Carlo tests. Journal of the American Statistical Association, 91, 1071-1078.

Brandstätter, E., and Kepler, J. (1999). Confidence intervals as an alternative to significance testing. Methods of Psychological Research Online, 4, 33-46.

Casella, G., and Berger, R. L. (2002). Statistical Inference (2nd ed.). Duxbury Press, Belmont, California.

Chachi, J., and Taheri, S. M. (2011). Fuzzy confidence intervals for mean of Gaussian fuzzy random variables. Expert Systems with Applications, 38, 5240-5244.

Colubi, A., and González-Rodríguez, G. (2007). Triangular fuzzification of random variables and power of distribution tests: empirical discussion. Computational Statistics and Data Analysis, 51, 4742-4750.

Colubi, A., González-Rodríguez, G., Lubiano, M. A., and Montenegro, M. (2006). Exploratory analysis of random variables based on fuzzification. In J. Lawry et al. (Eds.), Soft methods for integrated uncertainty modelling (p. 95-102). Berlin: Springer.

Dümbgen, L. (1998). New goodness-of-fit tests and their application to nonparametric confidence sets. The Annals of Statistics, 26, 288-314.

Feng, Y. (2000). Gaussian fuzzy random variables. Fuzzy Sets and Systems, 111, 325330.

Filzmoser, P., and Viertl, R. (2004). Testing hypotheses with fuzzy data: the fuzzy pvalue. Metrika, 59, 21-29.

Finner, H. (1994). Two-sided tests and one-sided confidence bounds. The Annals of Statistics, 22, 1502-1516.

González-Rodríguez, G., Colubi, A., and Gil, M. A. (2006a). A fuzzy representation of random variables: An operational tool in exploratory analysis and hypothesis testing. Computational Statistics and Data Analysis, 51, 163-176.

González-Rodríguez, G., Colubi, A., and Gil, M. A. (2012). Fuzzy data treated as functional data: A one-way ANOVA test approach. Computational Statistics and Data Analysis, 56, 943-955. 
González-Rodríguez, G., Montenegro, M., Colubi, A., and Gil, M. A. (2006b). Bootstrap techniques and fuzzy random variables: Synergy in hypothesis testing with fuzzy data. Fuzzy Sets and Systems, 157, 2608-2613.

Grzegorzewski, P. (2000). Testing statistical hypotheses with vague data. Fuzzy Sets and Systems, 112, 501-510.

Grzegorzewski, P. (2009). k-sample median test for vague data. International Journal of Intelligent Systems, 24, 529-539.

Grzegorzewski, P., and Hryniewicz, O. (1997). Testing hypotheses in fuzzy environment. Mathware and Soft Computing, 4, 203-217.

Holm, S. (1999). Multiple confidence sets based on stagewise tests. Journal of the American Statistical Association, 94, 489-495.

Hryniewicz, O. (2006). Possibilistic decisions and fuzzy statistical tests. Fuzzy Sets and Systems, 157.

Kruse, R., and Meyer, K. D. (1987). Statistics with Vague Data. Dordrecht: D. Reidel Publishing Company.

Lehmann, E. L., and Romano, J. P. (2005). Testing Statistical Hypotheses (3rd ed.). New York: Springer.

Mohammadi, J., and Taheri, S. M. (2004). Pedomodels fitting with fuzzy least squares regression. Iranian Journal of Fuzzy Systems, 1, 45-62.

Montenegro, M., Colubi, A., Casals, M. R., and Gil, M. A. (2004). Asymptotic and bootstrap techniques for testing the expected value of a fuzzy random variable. Metrika, 59, 31-49.

Mukerjee, R., and Reid, N. (2001). Comparison of test statistics via expected lengths of associated confidence intervals. Journal of Statistical Planning and Inference, 97, 141-151.

Nguyen, H. T. (2006). An Introduction to Random Sets. Boca Raton, FL: Chapman and Hall/CRC.

Parchami, A., Mashinchi, M., Yavari, A. R., and Maleki, H. R. (2005). Process capability indices as fuzzy numbers. Austrian Journal of Statistics, 34, 391-402.

Parchami, A., Taheri, S. M., and Mashinchi, M. (2010). Testing fuzzy hypotheses based on vague observations: a p-value approach. Statistical Papers, 51, 209-226.

Press, S. J. (1966). A confidence interval comparison of two test procedures proposed for the Behrens-Fisher problem. Journal of the American Statistical Association, 61, 454-466.

Puri, M. L., and Ralescu, D. A. (1985). The concept of normality for fuzzy random variables. The Annals of Probability, 13, 1373-1379.

Puri, M. L., and Ralescu, D. A. (1986). Fuzzy random variables. Journal of Mathematical Analysis and Applications, 114, 409-422.

Taheri, S. M. (2003). Trends in fuzzy statistics. Austrian Journal of Statistics, 32, 239257.

Tryon, W. W. (2001). Evaluating statistical difference equivalence, and indeterminacy using inferential confidence intervals: an integrated alternative method of conducting null hypothesis statistical tests. Psychological Methods, 6, 371-386.

Viertl, R. (2006). Univariate statistical analysis with fuzzy data. Computational Statistics and Data Analysis, 51, 133-147. 
Viertl, R. (2011). Statistical Methods for Fuzzy Data. Chichester: John Wiley and Sons.

Wu, H. C. (2005). Statistical hypotheses testing for fuzzy data. Information Sciences, $175,30-57$

$\mathrm{Wu}, \mathrm{H}$. C. (2009). Statistical confidence intervals for fuzzy data. Expert Systems with Applications, 36, 2670-26760.

Zimmermann, H. J. (2001). Fuzzy Set Theory and its Applications (4th ed.). Boston: Kluwer Academic Publishers.

Authors' addresses:

Jalal Chachi and Seyed Mahmoud Taheri

Department of Mathematical Sciences

Isfahan University of Technology

Isfahan 8415683111, Iran

E-mail: J.Chachi@math.iut.ac.ir, Taheri@cc.iut.ac.ir

Reinhard Viertl (Corresponding author)

Technische Universität Wien

Institut für Statistik und Wahrscheinlichkeitstheorie

Wiedner Hauptstraße 8-10/107

A-1040 Wien, Austria

E-mail: R.Viertl@tuwien.ac.at 